\title{
Die spezielle funktionelle Anpassung der Röhrenknochen-Diaphyse.
}

\author{
Von \\ Prof. Dr. F. A. M. W. Gebhardt, \\ Halle a. $S$.
}

Mit 13 Figuren im Text.

Eingegangen am 8. Mai 1910.

Im allgemeinen, wenn man lediglich Knochen vom Menschen mit sfunktionellen Gesichtspunkten untersucht, wird man leicht zu der Überzeugung kommen, daß die äußere Gestalt des Knochens etwas von außerhalb des Knochens gegebenes darstellt, gewissermaßen die rom "Bauherrn Vererbung" gestellte Aufgabe, deren Ausführung im einzelnen dann durch die besonderen Anpassungserscheinungen der inneren Struktur in zweckmäßiger Weise stattfindet.

Gerade diejenigen Forscher, welche fest überzengt von der hohen funktionellen Bedeutung der trajectoriellen Spongiosaarchitekturen sind, halten an der einheitlichen inneren funktionellen Struktur der äußerlich »unfunktionellen " Gesamtform besonders fest. Wiederholt finden sich da Äußerungen, daß die Spongiosa nur eine aufgeblätterte Compacta und die Compacta nur eine verdichtete Spongiosa sei. Es wird in diesen Fällen die kompacte Diaphyse der Röhrenknochen gewöhnlich mit besonđerer Emphase mit den Verdichtungen der Zugund Drucklinien am Rande des Knochens identifiziert.

Nun kann es aber einem aufmerksamen Beobachter, zumal wenn er sich nicht auf die Untersuchung menschlicher Knochen beschränkt, gar nicht entgehen, daß eine solche Auffassung für die vorhandenen Compactamassen in vielen Fällen ganz andre Grenzen verlangen würde, als sie sie tatsächlich darbieten. Vor allen Dingen ist es ja 
bei dieser Auffassung vollständig unzulässig, đaß eine compakte Diaphyse auch noch dort an Dicke zunimmt, wo keine Spongiosabälkchen (theoretisch gesprochen also keine Trajectorien) mehr in dieselbe einstrahlen. Gerade diese Dickenzunahme ohne lokale Spongiosaeinstrablung läßt sich aber an sehr vielen Knochen beobachten. Vielleicht am schönsten ist dies der Fall in den langen Extremitätenknochen der Wiederkäuer, aber auch des Pferdes, des Schweines and noch andrer größerer Huftiere. Wenn wir ein Femur oder eine Tibia rom Rind (Fig. 1) oder eine Tibia vom Pferd (Fig. 2) der Länge nach aufschneiden (am besten vergleicht man in praxi frontale

Fig. 1.

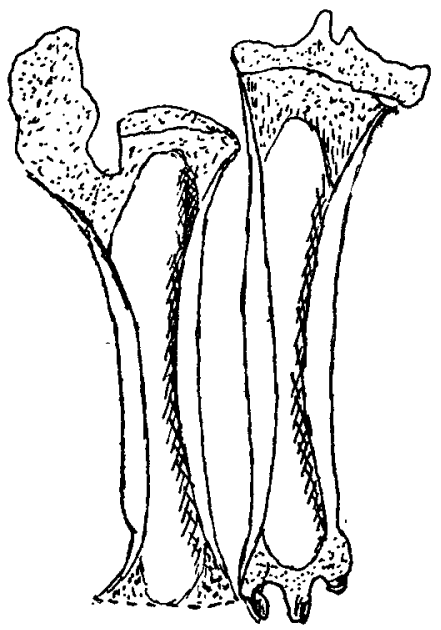

Fig. 2.

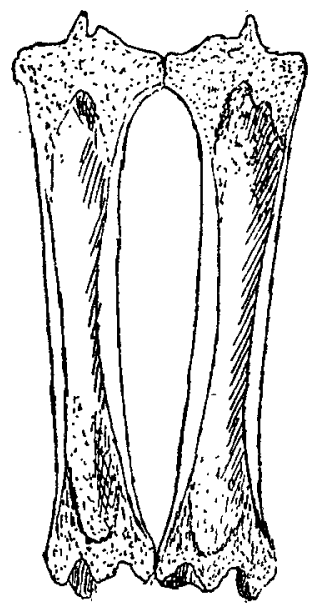

und sagittale Längsschnitte), so finden wir, beim Rind noch mehr wie beim Pferd, eine wesentlich nur auf die Knochenenden beschränkte Spongiosa, dabei aber eine gesetzmäßige Zunahme in der Wanddicke der dicken compakten Schaftröhre nach der Mitte der Länge hin. Genau dasselbe können wir, und zwar teilweise mit noch viel weitergehender Verdrängung der Spongiosa, auch an den andern langen Extremitätenröhrenknochen der größeren Wiederkäuer, ganz besonders gut ansgebildete Fälle an den Mittelhand- und Mittelfußknochen der großen wildlebenden (Antilopen, Giraffe!) Formen beobachten.

Hier herrscht also offenbar eine Unstimmigkeit, die sich nicht ohne weiteres mit den herrschenden Lehren über die funktionelle Struktur der Knochen in Einklang bringen läßt. Sehen wir weiter! 
Die schönsten trajectoriellen Fachwerkstrukturen selbst haben bei allen ihren Vorteilen auch einen gewichtigen Mangel. Die Vorteile gipfeln im wesentlichen darin, daß in den einzelnen Teilen, den statischen Stiitzelementen, eines trajectoriellen Fachwerkes, das Material im wesentlichen auf Zug und Druck und wenigstens primär nicht auf Schub beansprucht ist. Da die Schubbeanspruchung die meisten Materialien sehr viel widerstandsloser findet als die andern Beanspruchungen, so ergibt sich daraus die Möglichkeit, unter Benutzung trajectorieller Fachwerkarchitekturen mit erheblicher Materialersparnis zu bauen. Dabei haftet aber dem Fachwerk sowohl wie dem massiven Körper, namentlich bei längeren, auf Biegung oder Zerknickung beanspruchten Balken, noch der gemeinsame Übelstand an, daß dem Entstehen "gefährlicher Querschnitte«, d. h. der Anhäufung der Spannung bis zu bedroblicher Höhe an gewissen Stellen der Konstruktion, an sich nicht entgegengearbeitet ist.

Den meisten ïber trajectorielle Strukturen arbeitenden Biologen scheint dieser Nachteil des Fachwerks gar nicht bekannt zu sein. Ich zitiere deshalb aus dem berühmten Buche „Die graphische Statik * von Culmans diejenige Stelle, an der er von den Spannungsänderungen in den körperlich dargestellten Zug- und Drucklinien eines auf Biegung beanspruchten Balkens redet. „Die Spannung nimmt in ihnen zu, von dem Rand des Balkens, anf dem sie senkrecht stehen und wo sie den Druck oder Zug äußerer Kräfte aufnehmen können, gegen den Rand hin, mit dem sie parallel laufen.« Daraus folgt also, daß selbst in diesem sozusagen nur als trajectorielles Gerippe dargestellten Balken (genau so wie bei einem massiven unter gleichen Umständen, vgl. Fig. $3(x Q)$ an der Einmauerungsstelle ein $\gg$ gefährlicher Querschnitt existiert ${ }^{1}$. Ganz ebensowenig würde anch ein ohne Riucksicht hierauf gebautes, noch so fein ausgearbeitetes trajectorielles Spongiosafachwerk den Knochen vor der Entstehung gefährlicher Querschnitte schützen. Die Erfahrungen, welche wir in der Chirurgie über die Frakturen durch indirekte Gewalt gesammelt haben, sprechen durchaus dagegen, daß solche "gefährliche Quer-

1) Es würde sich dieser Übelstand allerdings durch eine gesetzmäßige Zunahme der Stützelementendicke nach dem $\gg$ gefährlichen Querschnitt" hin vermeiden lassen, wie in der Tat eine solche zweckmäBige Dickenabstufang der Banelemente (in angenäherter Form) in großen technischen Fachwerkbanten angewendet wird. In idealer Ansführung entstïnde dadurch dann ein Fachwerk s gleicher Festigkeit. (Vgl. unten iuber die sKörper gleicher Festigkeit 
schnitte $\mathrm{in} \mathrm{den} \mathrm{Knochen} \mathrm{in} \mathrm{typischer} \mathrm{Weise} \mathrm{vorhanden} \mathrm{sind.} \mathrm{Wäre}$ das nämlich der Fall, dann müßte jeder Knochen in viel typischerer Weise auch charakteristische Anhäufnngsstellen und Verlaufsweisen von Frakturen (durch indirekte Gewalt!) besitzen, vor allen Dingen aber, er müßte überhaupt viel zerbrechlicher sein, als es tatsächlich der Fall ist. Die aus den Versuchen von Meserer (Meserer, O., Über Elastizität und Festigkeit der menschlichen Knochen. Stuttgart 1880) sich ergebende sehr erhebliche absolute Bruchfestigkeit des Knochens, die Art, wie der Brach schließlich eintritt, und die namentlich von BARFURTH betonte anßerordentliche Geringfügigkeit der den Widerstand leistenden Masse stehen da in einem Gegensatz, welcher mit der Zulassung gefährlicher Querschnitte im unverein-

Fig. 3.

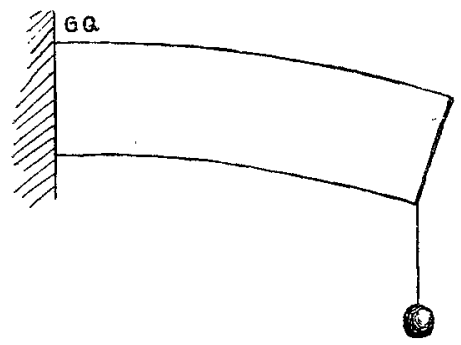

Fig. 4.

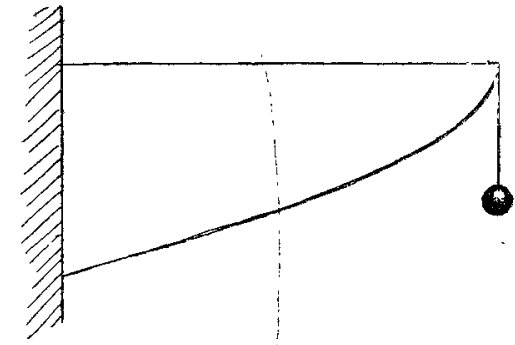

baren Widerspruche steht. Also auch hier wieder eine Unstimmigkeit, und zwar eine sogar quantitativ recht anffallende.

Nun gibt es in der Tat noch ein Mittel, durch welches sich die Widerstandsfähigkeit eines Körpers mit Rücksicht auf bestimmte Beanspruchungsweisen höher gestalten läßt, als die eines andern bei gleichem und selbst höherem. Materialaufwand. Ich brauche dazu z. B. bei einem gebogenen Balken nur die Querschnitte des Balkens von der Belastungs- nach der Einspannungsstelle so wachsen zu lassen, daB in keinem von ihnen die auf die Flächeneinheit entfallende Spannungsgrößen den Anfangswert an der Belastungsstelle überschreiten. Man bezeichnet einen solchen Körper als einen „Körper gleicher Festigkeit«. Für das gewählte Beispiel der Biegungsbeanspruchung eines an einem Ende eingemauerten Balkens würde sich z. B. in der Seitenansicht eine Zuschärfung des Balkens nach einer Parabel, bei lediglich in einer Richtung erfolgenden Biegung nach einer halben Parabel (Fig. 4) ergeben, wobei also, rechteckige Querschnitte angenommen, die Rechtecke bei konstan- 
ter Breite wie die Wurzeln der ganzen Zahlen in der Höhe zuzunehmen hätten, wenn man von der Belastungs- nach der Einmauerungsstelle zu fortschreitet. Sowohl für den gedachten wie auch für verwandte Beanspruchungsfälle (z. B. den auf Strebfestigkeit, Fig. 5) lassen sich eine ganze Reihe solcher Körper gleicher Festigkeit nach verschiedenen Prinzipien konstruieren, oder streng genommen unendlich viele, da sich das erwähnte Prinzip: Zunahme der Querschnitte mit dem Spannungswachstum derart, daß die Spannung für die Flächeneìnheit aller Querschnitte konstant bleibt, auf unendlich viele Weisen verwirklichen läßt (vgl. Recleaux, Der Kon-

Fig. 5.


strukteur. B Braunschweig 1895. Vieweg \& Sohn).

Halten wir uns aber zunächst einmal an Fig. 6. die in den Lehrbüchern der Festigkeitslehre gewählten Beispiele und beim Knochen an den relativ einfachen Fall der langen Extremitätenröhren!

Der uns interessierende Fall ist offenbar derjenige, wo es sich gleichzeitig um Körper gleicher Biegungs-, gleicher Streb-, gleicher Torsionsfestigkeit handelt. Handelt es sich da um einen an beiden Enden fixierten und in der Mitte beanspruchten Stab von massivem Querschnitt, so ergeben in relativ guter Übereinstimmung alle drei Beansprnchungsarten ziemlich ähnliche Gebilde, welche zwar quantitative Verschiedenheiten ihrer Abmessungen aufweisen, aber alle doch

immer ein Anwachsen der Querschnitte nach der Längenmitte hin zeigen (vgl. Fig. 6). Also erscheint offenbar unser Fall ziemlich hoffnungslos? Denn bekanntlich zeigen fast alle Knochen, die hier als Vergleich in Frage kommen könnten, eine Versehmälerung im Schaft und Verbreiterung nach den Enden zu. So habe ich in der Tat wiederholt urteilen hören! Dabei kann es einem aber bei der einfachsten Beruicksichtigung der tatsächlichen Verhältnisse gar nicht entgehen, daB hier in Wirklichkeit eine Analogie nicht besteht. Denn der Knochen hat eben keine massiven Querschnitte, seine Querschnitte sind sich auch keineswegs ähnlich, und seine Hohlräume haben sowohl im Längs- wie im Querschnitt eine keineswegs konstante oder auch nur relativ ähnliche Ausdehnung. Nun weiß aber 
Die spezielle funktionelle Anpassung der Röhrenknochen-Diaphyse. 521

jeder Ingenieur, daß bei einem nicht massiven Körper das Material auf dem Querschnitt eine ganz verschiedene Bedeutung für die Widerstandsfähigkeit des ganzen Gebildes hat, je nachdem es dem Querschnittsschwerpunkt näher oder ferner liegt, und wieder verschiedene, ob es bei gleicher Entfernung vom Querschnittsschwerpunkt in der Biegungsebene oder nur seitlich in der neutralen Schicht liegt, daB beispielsweise beim Vorhandensein einer Hauptbiegungsebene dieselbe Materialmenge in derselben Entfernung rom Querschnittsschwerpunkt das eine Mal sagen wir einen Widerstandswert $W$, das andre Mal einen Widerstandswert $W h^{2}$ z. B. gegen Biegung bedeuten kann, wenn sie nämlich um die Entfernung $h$ vom Schwerpunkt das eine Mal senkrecht zur Biegungsebene in der neutralen Schicht, das andre Mal in oder parallel der Biegungsebene von der »neutralen Schicht* entfernt liegt. Solange ein solcher Körper einigermaßen durch einfache mathematische Formen darstellbar ist, kann man sich rein rechnerisch ein Urteil über die in ihm vorhandenen Widerstandsverhältnisse bilden, bei unsern Objekten erscheint dies, weil eben einfach alles von vornherein als variabel eingeführt ist, zunächst völlig aussichtslos. Eins muß aber mit äußerster Bestimmtheit betont werden, daß in keiner Weise aus der äußeren Form des Knochens ein Schluß abgeleitet werden kann, der gegen seine Auffassung als Körper gleicher Festigkeit spricht.

Ganz im Gegenteil lassen sich für diese Auffassung selbst des erwachsenen Knochens anf anderm Wege in einer großen Anzahl von Fällen (und für den sich entwickelnden Knochen ỉberhaupt immer) eine Reihe gewichtiger Gründe vorbringen.

Zunächst ist es durchans nicht unfruchtbar, sich einmal mit dem Aussehen relativ einfacher, hohler $»$ Körper gleicher Festigkeit zu beschäftigen. Es erweist sich als praktisch, dabei zunächst den Fall der Strebfestigkeitsbeanspruchung anzunehmen, der ja auch im großen ganzen gut den natürlichen Beanspruchungsverbältnissen der langen Röhrenknochen entspricht. Ein voller Stab hat dabei eine Form, wie sie uns Fig. 6, etwas gestreckt (was theoretisch zulässig!) nach REULEauX kopiert, vorführt. Wir sehen hier einen Körper, welcher sich nach den Enden zu verdünnt, nach der Mitte zu verdickt. Offenbar steht gar nichts im Wege, auch ein cylindrisches Rohr (Fig. 7, welches uibrigens für Torsionsangriff nur am Ende ein Körper »gleicher Festigkeit« ist!) in ganz analoger Weise zu einem Körper gleicher Streb- usw. Festigkeit dadurch umzugestalten, daß ich seine Wanddicke nach der Mitte der Länge zu in ähnlicher Weise von beiden Seiten her zunehmen 
lasse (Fig. 8). Unter Beibehaltung der cylindrischen Bohrung geschieht das in der Technik sogar außerordentlich oft, ganz besonders auch für Formen gleicher Biegungs- und Torsionsfestigkeit, wie sie z. B. die Tragachsen schwerer Schwung- und Wasserräder darstellen.

Fiv 7.

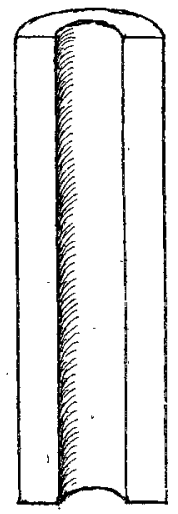

Fig. 8.

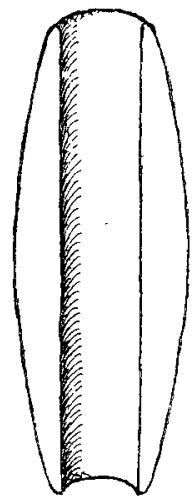

Fig. 9.

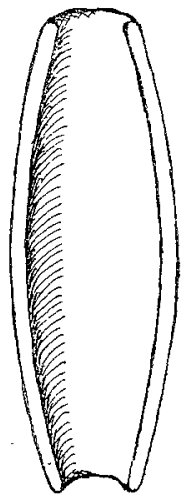

Derartige Körper werden sogar mit besonderer Vorliebe als Rechenbeispiele in den Lehrbüchern verwendet. Man kommt dabei wegen der Vorteile des Hoblbauens an sich mit relativ geringem Materialaufwande aus. Dasselbe gilt von entsprechenden hohlen Tragesänlen.

Fig. 10.

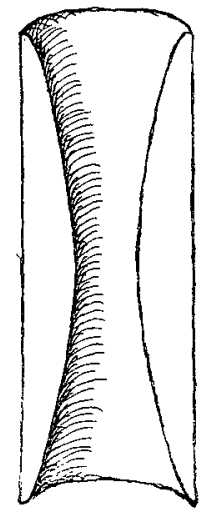

Fig. 11.

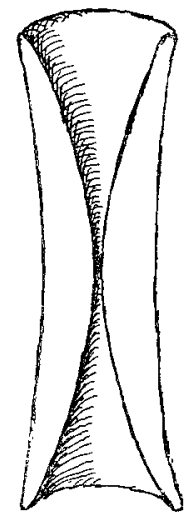

Ja es zeigt sich, eben weil das Material bei größerer Entfernung von der Schwerlinie (d. h. der die Querschnittsschwerpunkte verbindenden Linie) eine verschiedene, und zwar höhere Bedeutung für die Widerstandsfähigkeit hat, daß selbst bei gleichbleibender Wanddicke durch eine entsprechende Wandwölbung schon Annäherndes für gewisse Beanspruchungen $\mathrm{zu}$ erreichen ist (Figur 9). Nun liegt aber offenbar auch nicht das geringste Hindernis vor, die Dickenzunahme, von beiden Enden nach der Längenmitte zu, statt auf der Außenseite auf der Innenseite des ursprünglich eylindrisch gedachten Rohres stattfinden zu lassen (Fig. 10). Da das Material hierbei nicht ganz so gut auf seine Widerstandsfähigkeit ausgenützt wird, so wird sich 
Die spezielle funktionelle Anpassung der Röhrenknochen-Diaphyse. 523

freilich dabei etwas größerer Materialaufwand als nötig erweisen, als bei der Verdickung auf der Außenseite, und wenn endlich überhaupt das ganze Rohr aus irgend einem Grunde nach den Enden zu eine Erweiterung zeigen soll, so ist auch das noch in keiner Weise ein Hindernis, auf die geschilderte Art und Weise einen Körper gleicher Festigkeit herzustellen, freilich wieder mit etwas mehr Materialaufwand (Fig. 11). Es können aber alle diese größeren Materialaufwendungen durch eine Gesamterweiterung des ganzen Rohres auch wieder unnötig gemacht werden. Mit unserm letzten Beispiel sind wir aber schon vollständig in den Formenkreis gekommen, welchem die Diaphysen fast sämtlicher langer Röhrenknochen angehören. Daß das Mittel der absoluten Querschnittserweiterung viel verwendet wird, ist unter anderm Gesichtspunkt bekannt.

Jetzt gewinnt die Verdickung der Wand in Röhrenknochenschäften nach der Mitte der Länge zu mit einem Schlage eine ganz besondere und andre Bedeutung, als die, lediglich der körperliche Ausdruck der Verdichtung der Zug- und Drucklinien zu sein, namentlich wenn man sich überzeugt, daß diese gesetzmäßige Dickenzunahme um so schöner und deutlicher zu sehen ist, je stärker die Reduction der Spongiosaarchitektur in den betreffenden Knochen ist, und wenn man daran denkt, daß dieses Verhalten ihrer Wanddicke von Anfang an in der Entwicklung vorhanden ist, ehe von geordneten, ja überhaupt von irgend welchen Spongiosabildungen die Rede ist. Mit andern Worten, es zeigt die Diaphyse der langen Röhrenknochen ein Verhalten, welches sie obne weiteres zu einem $»$ Körper gleicher Festigkeit " bei Beanspruchung auf Strebfestigkeit, auf Biegungsfestigkeit und auf Torsion stempelt.

Hier muß gleich ausdrücklich betont werden, daß für diese Auffassung überhaupt lediglich das Vorhandensein einer einigermaßen gesetzmäßigen Dickenzunahme der compakten Wand von den Enden nach der Mitte zu entscheidend ist, und daB es keineswegs anf den zahlenmäßigen Nachweis eines ganz bestimmten Modus dieser Dickenzunahme ankommt. Dies ist schon einfach aus dem Grunde nicht möglich, weil es sich ja um außerordentlich wechselnde Beanspruchungen handelt, und weil für jede einzelne von diesen (und sogar schon für wechselnde Stärke der Beanspruchung, indem mit dieser die absoluten dimensionalen Abmessungen schwanken) die ideale Form etwas anders ausfällt. Sie haben eben alle nur das Gemeinsame der überhaupt gesetzmäßigen Wanddickenzunahme von den Röhrenenden nach der Röhrenmitte zu, und es dürfte die wirklich 
zar Ausbildung gelangende Form im wesentlicben in ibren Konturen den äußeren Umriß aller dieser übereinandergelegten, übrigẹs immerhin untereinander recht ähnlichen, Einzellösungen der jeweils vorliegenden Aufgabe darstellen. Auch hier bedingt im übrigen schon die notwendige "Sicherheit «, d. h. die Notwendigkeit, die Ausführung: um ein Mehrfaches stärker geschehen zu lassen, als der stärksten mutmaßlichen Beanspruchung entspricht (vgl. TrIEPEL, Die Stoßfestigkeit der Knochen. Arch. f. Anatomie u. Physiologie. 1900), daß die gedachten Schwierigkeiten und Unstimmigkeiten in Wirklichkeit gar nicht zur Entstehung gelangen. Man kann im Gegenteil erkennen, und zwar an sehr vielen Beispielen, daß auch hier wieder der vom Kleinen ins Große bauende Knochen viel vollkommener den speziellen Verhältnissen Rechnung zu tragen vermag, als das in der Technik zu geschehen pflegt, indem z. B. eine Hauptdruckseite im ganzen stärker ausgeführt erscheint $\mathfrak{n}$. dgl. $\mathrm{m}$.

Dazu ist noch etwas aus der Technik in unserm Sinne anzuführen. An die strenge Ausführung der bei verschiedenen Beanspruchungen möglichen und üblichen Körper gleicher Festigkeit fühlt sich auch der Techniker fast nie gebunden, denn die wesentlichen Vorteile dieser Formen werden zum größten Teil auch bei rohen Annäherungen erreicht. Es würde auch in der Tat niemals einem Techniker in den Sinn kommen, das in diesem Sinne Vorteilhafte der Massenverteilung in den Röhrenknochenschäften zu bestreiten. Anderseits läßt sich bei einer Betrachtung irgendwelcher technischer, namentlich Maschinenbauten gar nicht verkennen, daß die moderne Zeit vor der älteren sich dadurch auszeichnet, daß, wenigstens bei guten Konstrukteuren, diese Formen gleicher Festigkeit die ganze Formgebung geradezu beherrschen, sich gewissermaßen zu den wesentlichen Bestandteilen eines Baustiles anfgeschwungen haben. Höchst charakteristisch ist dafür, und noch mehr für die Freiheit in Gebrauch und Auffassung der Körper gleicher Festigkeit, eine Stelle in ReuleauX »Konstrukteur «, die ich deshalb zitieren möchte. "Körperformen von gleicher Festigkeit ergeben sich, wenn man in einem Körper die Quersehnitte so wählt, daß in allen die größte Spannung $S$ denselben Wert erhält, wodurch eine verhältnismäßig sehr guinstige Materialverwendung erzielt werden kann. Solche Körperformen werden indessen gewöhnlich nicht mit Genauigkeit, sondern nur näherungsweise zur Ansführung gebracht; sie dienen in vielen Fällen sogar nur als stilistische Grundformen, die einer Konstruktion nur den Ausdruck der gleichen Festigkeit zu verleihen haben, obne daß 
Die spezielle funktionelle Anpassung der Röhrenknochen-Diaphyse. 525

diese streng eingehalten würde. Dem Konstrukteur sind sie ans beiden Grïnden von Wert; ja es möchte der Zahl nach die zweite Benutzungsart, die des stilistischen Vorbildes, häufiger sein als die erstere. Sind dem Konstruierenden die Festigkeitsformen recht geläufig geworden, und hat er sich frei gemacht von dem Gedanken, daß die für die Gleichhaltung der Festigkeit erforderlichen Kurven streng eingehalten werden $m \ddot{u} ß t e n$, so wird er bald dahin gelangen, stets ansprechende gefällige Linien für schwer zu belastende Konstruktionsteile zu finden, indem er die Grundform, welche der starre mathematische Ausdruck liefert, mit künstlerischer Freiheit und Selbständigkeit in das Leben überträgt. "

Nun ist aber das schönste an dieser ganzen Auffassung des Verhaltens der Diaphysenwanddicke, daß dabei nicht, wie leider sonst oft, die causalen Verhältnisse für die Entstehung vollständig im Dunkeln liegen. Aber sie sind nicht nur, wie wir gleich sehen werden, eine Erklärung für die ohne weiteres hier durch Augenschein zu erhebenden tatsächlichen Verhältnisse, sondern sie machen es auch außerordentlich wahrscheinlich, daß das Prinzip des Körpers gleicher Festigkeit hier in einer viel vollkommeneren Anwendungsform vorliegt, als man vielleicht beim ersten Anblick glauben möchte, und sie geben noch überdies das Material her für die Ausfüllung einer höchst empfindlichen Lücke in unsern causalen Vorstellungen von dem $\mathrm{Zu}$ sammenhange der funktionellen Anordnungen der feinsten Bauelemente mit dem funktionellen Gesamtbauplane des ganzen Körpers.

Die fraglichen causalen Verhältnisse dürften nämlich bereits in der frühen Entwicklung des Knochens die ausschlaggebende Rolle spielen. Es war mir eine sehr große Freude, in der vergleichenden Anatomie von Gegenbaur ein Schema vorzufinden, welches ohne weiteres zur Demonstration der hier in Frage kommenden mechanischen Verhältnisse benutzt werden kann (vgl. Fig. 12). Daneben steht $\gg$ Es ist beachtenswert, daß die erste periostale Knochenbildung mehr oder minder in der Mitte der Länge erscheint, da wo der Skeletteil, denkt Fig. 12.
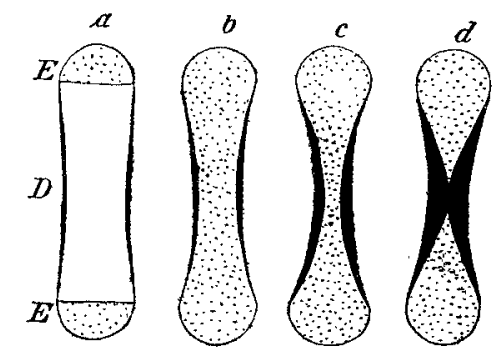

Aus Gegenbaur, Vergleichende Anatomie. man ihn sich z. B. als Hebelarm wirkend, den größten Widerstand zu bieten hat. Der vom Knochen umscheidete Knorpel muß auf dieser 
Strecke sein Wachstum sistieren. Da er hier auch die Stützfunktion verlor, befindet er sich in einem, Veränderungen seiner Struktur erklärenden Zustande«. Teh möchte dazu noch gleich auf die geradezu idealen Dickenverhältnisse des schwarz gezeichneten Knochenrohres aufmerksam machen, bezüglich deren jede einzelne Figur den Längsschnitt eines Rohres gleicher Festigkeit darstellt. Daß diese hier gezeichneten Verhältnisse in der Tat in den Röhrenknochendiaphysen sehr vieler Tiere eine deutliche Ausprägung erfahren, davon kann man sich ohne weiteres überzeugen, wenn man die Knochen der Amphibien, der Krokodile und sehr vieler andrer Reptilien auf

Eig. 13.

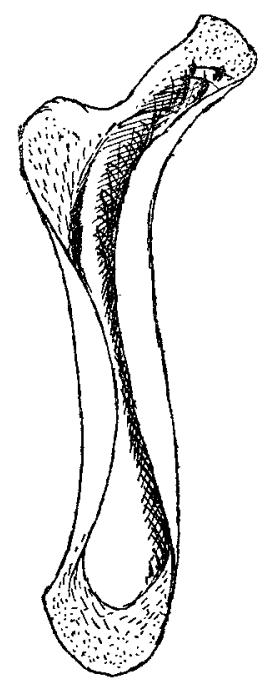
Längsschnitten ansieht. Die Verdickung in der Längenmitte bis zur völligen Verdrängung der Markhöhle zeigt ein Oberarm vom Krokodil sehr schön, welcher sich in meinem Besitz befindet, wie überhaupt gerade die Knochen der Krokodile, wohl wegen der vorwiegenden Biegungsund Torsionsbeanspruchung, recht gute Demonstrationsobjekte für die hier wesentlichen Wanddickenverhältnisse darstellen ( $r g l$. auch das Femur rom Krokodil Fig. 13). Kehren wir aber zu dem knorpligen Vorstadium des Röhrenknochens, und zwar in der Ontogense, zuriick, so hat den Gedanken der größten Spannungsanhäufung in der Längenmitte in neuerer Zeit besonders Tнома (R. Thoma, Synostosis suturae sagittalis cranii usw. Virchows Archiv. 188. Heft 2. 1907) als Entstehungsursache des periostalen Knochenringes verwertet, und zwar unter Eingehen auf verschie-

dene Beanspruchungsarten. Wir werden dem noch einiges hinzuzufügen haben. Vorher muß aber noch erwähnt werden, daß Roux für die Lokalisierung der Knochenbildung sowohl in der Epiphyse wie in der Diaphyse den lokalen Mangel an Abscherungsbeanspruchung verantwortlich macht (Ges. Abhandlungen. I. S. 810; II. S. 228).

Zu den von Thома angefuhrten Momenten ist noch einiges hinzuzufügen. Zunächst ist zu sagen, daß anch durch die typische Torsionsbeanspruchung in ganz gleicher Weise wie bei Zug, Druck, Biegung und Beanspruchung auf Strebfestigkeit mit einer gewissen Häufung in der Mitte der Länge der Röhrenkochenanlage, und zwar auch wieder in der periphersten Schicht, Spannungsmaxima entstehen, wie sich an der Hand der Festigkeitslehre ohne Mühe zeigen läßt. 
Dann ist aber auch die unter dem Einfluß der Beanspruchnng wirklich erfolgende Deformation der elastischen Knorpelanlage selbst in gleichem Sinne wirksam. Denn gerade so wie aus einem elastischen relativ kurzen Cylinder bei axialer Längsdruck-Beanspruchung eine Tonnenform entsteht, so erleidet anch die arspriinglich in der Mitte noch nicht eingeschnürte kurze Knorpelanlage hier eine elastische Verdickung und überhaupt Formveränderungen, welche immer in der Mitte der Länge am stärksten ausfallen. Zerrungen zwischen dem Knorpel und der ihn bedeckenden Faserhülle, dem Perichondrium, fallen hier gleichfalls somit am stärksten aus, so daß in jeder Hinsicht eine direkte Reizung zur periostalen Knochenbildung hier znnächst ihren größten Betrag erreichen muß. Alle diese Momente schaffen also eine Prädilektionsstelle für die Knochenbildung und sie schaffen auch sofort diese Knochenbildung in der Gestalt einer » Röhre gleicher Festigkeit", indem eben immer die Einflüsse in der Mitte der Knochenlänge am stärksten ausfallen. Dadurch wird eben von vornherein diese Mitte am stärksten angelegt, aber sie wird auch dann von selbst, da sie eben die zeitlich erste Anlage und alle späteren enthält, ihren Vorsprung vor den weiter nach den Enden zu gelegenen immer späteren Anlagerungen obne weiteres behalten. Sehr bald werden aber noch weitere Momente dazu kommen, welche eine geradezu ideale Herstellang eines Körpers gleicher Festigkeit aus dem periostalen Knochenrohr garantieren.

Sowie nämlich einmal der knöcherne Ring um die Diaphyse sich gebildet hat, übt er auch sofort seinerseits einen ausschlaggebenden Einfluß auf die Weiterbildung des ganzen Skeletteils aus. Das Deformationsbestreben des viel elastischeren, weicheren Knorpels unter dem Einfluß der Beanspruchung findet in seinem Bereich einen absoluten Widerstand. Damit wird auch der Seitenkontur, z. B. bei axialer Längsdruckbelastung, ein andrer. Statt wie früher in der Deformations»tonne « der Gleichung der elastischen Linie im ganzen zu folgen, erleidet er jetzt eine geradlinige Unterbrechung im Bereiche des knöchernen Ringes und wölbt sich dafür an den über und unter diesem gelegenen noch freien Knorpelpartien mit um so plötzlicherem Richtungswechsel hervor, sich mit vorspringendem Wulst auf die benachbarte Zuschärfung des Diaphysenringes stïtzend und sich auch wohl tiber dieselbe nach außen herüber wölbend. Wir haben mehrfache Gelegenheit, die Folgen dieses mechanischen gegenseitigen Verhältnisses zu beobachten. Erstens kommt es hier zu sehr intensiven Pressungen und Zerrungen der skeletbildenden Faserbülle, die 
an dieser Stelle ja gerade aus dem Periost zum Perichondrium wird. Sie bildet an dieser Stelle daher auch einen besonders widerstandsfähigen, die Basis des noch unbekleideten Knorpels circulär einschnürenden dicken Ring, die Ranviersche sencoche». Somit läßt sich diese auffällige und wichtige Stelle, welehe bei der späteren Entwicklung der Röhrenknochen bekanntlich eine wichtige Rolle zu spielen hat, in ihrer ersten Entstehung mit primitiven mechanischen Verhältnissen in Verbindung bringen. Eine weitere Folge des Aufstauchens der Knorpelenden auf die scharfe Kante des Diaphysenringes können wir bei Tieren beobachten, bei welchen, wie bei den Amphibien, sehr primitive Verhältnisse bestehen bleiben. So sehen wir z. B. beim Froseh sich das knorplige Ende der Röhrenknochen ïber den scharfen Rand der hohlen, knöchernen Diaphyse »mützenartig oder wie einen Stockknopf . . . herüberstülpen, und auf der äußeren Fläche der Diaphyse mit ziemlich scharfer Grenze aufhören \& (ECKER-GaupP, Anatomie des Frosches. 1. Band [in beiden Auflagen]). In mannigfacher Weise prägen sich dabei sowobl in der Richtung der Knorpelzellen wie auch in der lokalen Verteilung der histologischen Weiterentwicklung des Knorpels zu "Kalkknorpel" (GAUPP) bzw. »-Knochen" die mechanischen Beanspruchungsverhältnisse mehr oder weniger deutlich aus. Schon Ecker sagt in der ersten Auflage: »Der Knochencylinder der Diaphyse . . . ist gegen die Epiphyse durch hyalinen Knorpel ... abgeschlossen, dessen Zellen zwischen den Enden des Knochens ... in querer Richtung gelagert sind." Man sieht außerdem auch in der dort beigegebenen Figur, daß sich, soweit der hyaline Knorpel reicht, das Diaphysenende inwendig mit einer Schicht »Kalkknorpel " belegt hat, welche ihrerseits die Trichterform des Diaphysenendes noch verstärkt. Ich kann dem noch hinzufügen, daß sich in den Oberschenkelenden der ausgewachsenen großen Hallenser Rana esculenta eine förmliche Gewölbekonstruktion aus "Kalkknorpel " und stellenweise wohl bereits echtem Knochen als Abschluß des oberen Diaphysenendes entwickeln kann, welche von den erwähnten inneren Kalkknorpelauflagerungen ihren Anfang nimmt, nach dem Knochenende zu convex ist und schließlich nur noch in der Knochenachse eine kleine Durchbohrung zeigt. Die mikroskopischen Bilder zeigen hier eine so genaue Übereinstimmang der vorhandenen Strukturen mit dem Verlauf der gegenseitigen Reanspruchungsspannungen in Epiphyse und Diaphyse, daß dieses Objekt wohl eines genaueren Studiums wert ist, dessen Resultate ich an anderm Orte zu veröffentlichen beabsichtige. Hier nur soviel davon, 
Die spezielle funktionelle Anpassung der Röhrenknochen-Diaphyse. 529

daß sowobl aus der Richtung der Knorpelzellen in den einzelnen Bezirken wie auch aus der lokalen Auordnung dieser Weiterfortbildungen des Knorpelgewebes mit Sicherheit hervorgeht, daß ein Anstützen des Knorpelpfropfes an die Diaphysenwand von innen her in ähnlicher Weise stattfindet, wie sich ein Korkstöpsel oder ein Faßspund an die umfassende Wandung ankeilt.

Dieses » Einkeilen « der knorpligen Enden in den DiaphysenHohlcylinder findet natürlich auch bei den knorpligen Röhrenknochenanlagen der Säugetiere statt, allerdings ohne wesentliches äußeres Übergreifen iiber die Diaphysenkante und auch mit nur sehr wenig. weitem Eindringen des Knorpels in dieselbe in den späteren Stadien. Auch ist die Reaktion in bezug auf die histologische Weiterentwicklung des Knorpels eine ganz analoge. Auch hier entstehen, quer zum Diaphysenringende verteilt, endochondrale Knochenbildungen und weiter innerhalb des Diaphysenendes eine vollkommene Entlastungszone, da alle Beanspruchungen seitlich von den Knorpelenden auf den steifen Diaphysenhohlcylinder übertragen werden. Diese vollständige Entlastung innerhalb der knöchernen Diaphyse ist dann die Ursache für den vollständigen Schwund des Knorpels an dieser Stelle und Ersatz desselben durch Markgewebe (vgl. oben auch Gegenbaur!). Inzwischen geht der Anbau an die knöcherne Diaphysenanlage außen fortwährend weiter. Jede neue Lamelle, die sich außen auflagert, ist länger wie die vorhergehende und ihr Ende gleichzeitig auch immer etwas weiter, auch wieder wegen der erwähnten mechanischen Wechselbeziehung zwischen der knorpligen Epiphyse und dem knöchernen Diaphysenende, weil der Knorpel beim Aufstauchen nach anßen über den Rand strebt, und weil die Knorpelepiphyse selbst konstant durch Knorpelwachstum an Größe zunimmt. Dadurch kommt sehr rasch die »doppelkegelförmige «, mit "sanduhrförmiger * Höhlung versehene Gestalt der knöchernen Diaphyse zustande. Sehr bald beteiligen sich an der Formierung dieser Gestalt außer den äußeren Appositionsvorgängen auch innere Resorptionsvorgänge. Wir wissen nun gerade sehr genan aus der Untersuchung der viel späteren trajectoriellen Strukturen, namentlich mit Bezug auf die oft wunderbar deutliche Herausarbeitung derselben im Greisenalter, wie empfindlich die Resorptionsvorgänge auf den funktionellen Reiz reagieren (vgl. Rouxs »dimensionale Atrophie und Hypertrophie", besonders auch die "hypertrophische Atrophie usw.). Es ist somit eigentlich ganz selbstrerständlich, daß von diesen innen am stärksten vorhandenen Resorptionsvorgängen um so mehr von der Diaphysendicke verschont 
bleibt, je mehr wir uns der Längenmitte des Knochens nähern, weil in dieser Gegend eine Anhäufung sämtlicher Spannungen und damit die stärkste Beanspruchung des vorhandenen Materials stattfindet. Diese Gestalt der Diaphysencompacta, welche somit von Anfang an einen Körper gleicher Festigkeit repräsentiert (vgl. oben), bleibt nun lange Zeit erhalten, ziemlich unbekümmert darum, inwieweit außerdem noch im Innern des Knochens Spongiosa zur Ausbildung gelangt. Wir sehen sie in den reichlich spongiösen Knochen älterer menschlieher Föten, aber ebensowohl in den fast spongiosalosen Handwurzelund Fußwurzelknochen und Phalangen der Huftiere. (Ich möchte hier ausdritcklich darauf aufmerksam machen, daß gelegentlich schon makroskopisch, besonders aber mikroskopisch, wo man nur einen einzigen ebenen schnitt als Beobachtungsobjekt vor sich hat, hier und da eine Verwischung dieser Diaphysengestalt dadurch zustande kommen kann, daß sich gerade in der Schnittebene eine größere Anzahl von Gefäßräumen in unregelmäßiger Form und Verteilung im Diaphysengebiet breit machen. Der Sagittalschnitt der Femora trifft z. B. ziemlich sicher das Foramen nutritium längs! Es genügt in der Regel schon die Durchmusterung eines oder einiger benachbarter Schnitte, um die dadurch eventuell hervorgerufene Täuschung tiber die Diaphysenform zu eliminieren.)

Ist diese Auffassung von den mechanischen Verhältnissen und von deren Funktion als » dimensionaler gestaltender Reiz « (Roux) die richtige, dann müssen sich dafür auch noch besondere morphologische Stïtzen finden lassen. Das ist auch in der Tat der Fall. Diejenigen Spannungen, welche das Material am allerstärksten beanspruchen, die also auch dann noch wirksam sind, wenn die andern Spannungen mit der zunehmenden Menge des widerstandsfähigen Materials auf eine als gestaltender Reiz nicht mehr wirksame Größe herabgesunken sind, sind die Schubspannungen. Diejenige Beanspruchungsart, bei der sie vor allen andern primär auftreten, ist die Torsion. Wir dürfen deshalb erwarten, Spuren der Torsion an der Diaphyse ausgeprägt zu finden. Dies ist nun in der Tat in mannigfacher Weise der Fall (wobei aber die entstehenden Bildungen die bei der Torsion sekundären Zug- und Druckspannungen zum Ausdruck bringen, welche im Gegensatz zu den Schubspannungen positiv gestaltend oder konservierend wirken!).

Zum besseren Verständnis ist es nötig, vorauszuschicken, daß sich die Diaphyse in ihrer Rolle als »Körper gleicher Festigkeit « im wesentlichen bis zum knöchernen Festwachsen der Epiphysen erhält. 
Die spezielle funktionelle Anpassung der Röhrenknochen-Diaphyse. 531

Weiter unten soll hierauf mit ein paar Worten noch nähẹr eingegangen werden. Man findet nun in stark auf Torsion beanspruchten Knochen im Innern der Diaphyse an der Wand teilweise schöne Bälkchensysteme von der Art der trajectoriellen Bälkchenspongiosa, welche in der Richtung der maximalen Zug- und Druckspannungen bei der Torsion, d. h. unter gegenseitiger rechtwinkliger Kreuzung von beiden Seiten schräg gegen die Knochenlängsachse, angeordnet sind. Später vereinigen sich diese inneren Torsionsstrukturen vollständig mit der trajectoriellen Architektur des Knochens, wenn dieselbe eine Biegnngsarchitektur ist, dergestalt, daß die Biegungstrajectorien in ihrer dritten Dimension obne weiteres in die Torsionsstruktur im Bereiche des Schaftes übergehen. (Jeder, event. etwas ausgelöffelte, Längsschnitt eines Humerus oder Femur kann ohne weiteres durch Augenschein von der Richtigkeit dieser Behauptung überzeugen.) Statt dieser Torsionsbälkchenstrukturen feinerer Art können auch innerhalb der Diaphyse dünnwandige konzentrische Knochenröhren in größerer oder geringerer Ausdehnung vorhanden sein, die sich stellenweise in Torsionsbälkchensysteme auflösen. (Auch hierfuir geben viele menschliche Oberschenkelbeine Beispiele.)

Die ganze Art dieser Strukturen kennzeichnet sie ziemlich deutlich als durch Resorptionsvorgänge herausgearbeitet, und in der Tat sind ja ïberwiegend Resorptionsvorgänge im Innern der Diaphyse tätig, wenn auch das, was sie geschaffen haben, schließlich durch Apposition mit einer dünneren oder dickeren Generallamelle zum Schluß iiberkleidet werden kann. Noch viel deutlicher als hier ist diese iuberwiegende Rolle der Resorption an den ganz groben Torsionsbalken, wie sie im Innern des Humerus der großen Wiederkäner, des Femur vom Känguruh u. v. a. 0. durch die Resorption - herausgeschnitzt « werden. Ich habe schon in einer früheren Arbeit $\left.{ }^{1}\right)$ die mikroskopische Struktur dieser groben, von v. RecklinghaUsen und Roux bereits beobachteten und von letzterem auch bereits als Torsionsstruktur gedeuteten Balken ausführlich behandelt. Ich zeigte daselbst, daß sie in der Tat ohne jede eigne Struktur wirklich wie einfach aus der win toto konzentrisch" gebauten Diaphysenwand rein resorptiv heransgeschnitzt sind, indem ihre Konturen die Bauelemente der Diaphyse rüeksichtslos durchschneiden. Später, wenn das ganze Wachstum des Knochens einigermaßen zur Ruhe gekommen ist, legt

1) W. Gebhardi, Über funktionell wichtige Anordnungsweisen der gröberen und feineren Bauelemente des Wirbeltierknochens. I. Allgemeiner Teil. Arch. f. Entw.-Mechanik. Bd. XI u. XII. 1901. 
sich darüber ohne jeden Umbau eine innere ganz gleichmäßig dicke Generallamelle. So entbehren diese groben Balken jedes eignen feineren funktionellen Baues (vgl. 1. c. Fig. 34, 40-43 Tafel XVII u. XVIII) und kennzeichnerr sich somit absolut untriiglich als lediglich durch Resorption unter dem lokal konservierenden Einflusse der Torsionszug- und -druckspannungen gewonnen.

Somit müssen wir also auch erwarten, daß wir auf der Außenseite der Diaphyse Appositionsstrukturen durch Torsion, wenigstens unter besonders günstigen Umständen, zu sehen bekommen werden. Nun, solange der Knochen noch jugendlich ist, ist im Grunde genommen der ganze »in toto konzentrische "Aufbau der körperliche Ausdruck einer Reaktion auf Biegungs- und Torsionseinflüsse, wenigstens bei Humerus, Femur, Hand- und Fußwurzelknochen usw. der meisten Vierfüßler. Wenn der Knochen älter wird, dürfen wir anderseits aus verschiedenen Gründen nicht ohne weiteres mehr ausgedehnte Appositionsvorgänge an der Oberfläche erwarten. Auch die Generallamelle ist eine viel zu langsame Bildung, um, abgesehen von ihrer tatsächlich meist abwechselnd schräg gegen die Knochenlängsachse gekreuzten Lamellenfaserrichtung, deutliche grobe Torsionsstrukturen auftreten zu lassen. Das ändert sich sofort bei produktiven Knochenhautentzündungen. Ich verdanke Herrn Geheimrat Roux die Möglichkeit, an einem Humerus mit einer jedenfalls syphilitischen Periostitis in der Tat das Auftreten einer prachtrollen äußeren Torsionsstruktur im ganzen unteren Drittel konstatieren zu können. (Demonstriert auf dem Anatomenkongreß in Halle 1902 als Objekt und als Glasbild.)

Man sieht also, daß in der Tat die das Material am stärksten angreifende Beanspruchung, die Torsion, innen und außen ihre Ausprägung erhält, und zwar in genau mit den Postulaten Rovxs übereinstimmender Weise, indem ihre Schubspannungen resorptiv, ihre Zug- und Druckspannungen konservierend and positiv die Anbildung befördernd wirken.

Das alles führt aber zu der Überzeugung, daß auch auf allen Entwicklungsstufen der wachsenden Diaphysenröhre das innere Wegnehmen und das äußere Anbilden sich genau nach den vorhandenen Beanspruchungsverhältnissen richten. Es läßt sich diese Ansicht in der Tat noch durch das weitere Merkmal bekräftigen, daß auch bei den wachsenden röhrenförmigen Diaphysen die stärker beanspruchte Druckseite dicker ausgebildet wird, ohne daß dabei im ganzen die Form gleicher Festigkeit verloren geht (vgl. Femora, Humeri und Tibien älterer menschlicher Föten, ferner Metacarpalien, Metatarsalien 
Die spezielle funktionelle Anpassang der Röhrenknochen-Diaphyse. 533

und Phalangen bis zum erwachsenen Zustande). Damit aber ist ohne weiteres ausgesprochen, dab hier die Herstellung eines Körpers gleicher Festigkeit in geradezu idealer Weise garantiert erscheint, indem es die Spannungen selbst sind, die lokal regulatorisch die Appositions- und Resorptionsvorgänge beeinflussen.

Es bleibt uns noch die Frage zu erörtern, in welchem Verhältnis die somit in einem großen und wichtigen Abschnitt der Entwicklung, nämlich von der knorpligen Anlage an bis zur knöchernen Verschmelzung der Epiphysen wesentlich nach dem Prinzip der » Körper gleicher Festigkeit" mit besonderen Vorteilen gegenüber mechanischen Beanspruchungen gebaute Diaphyse zu den definitiven trajectoriellen Strukturen steht. Einiges geht ohne weiteres aus den vorhergehenden Bemerkungen hervor und läßt sich auch ohne weiteres durch Augenschein an jugendlichen menschlichen Knochen erhärten. Erstens nämlich ist der Sitz für die Entstehung der trajectoriellen Strukturen wesentlich die Stelle, an welcher sich die Epiphyse von innen her an das Diaphysenrohr anstïtzt. Damit dürfte auch ohne weiteres die Erklärung gegeben sein, weshalb gerade die schräg nach der gegenüberliegenden Wand verstrebten Biegungsarchitekturen sich so außerordentlich häufig entwickeln, selbst in Knochen, deren dimensionale Entwicklung und Beanspruchungsart auch mit einer einfacheren Architektur gut auskommen könnte. Ferner haben wir oben gesehen, daß mit der gesehilderten Ausbildung der Diaphyse gleichzeitig eine gewisse Tendenz zur Ausbildnng von Torsionsstrukturen vorhanden ist, und daraus erklärt sich wieder, weshalb die so häufigen Biegung:sarchitekturen mit besonderer Vorliebe im Schaft nach der Art von Torsionsstrukturen auslaufen. Also finden hier gerade ungezwungen zwei Punkte ihre Erklärung, die einer solchen bisher recht unzugänglich waren.

Bezüglich des zeitlichen Inbeziehungtretens der beiden Banprinzipien muß zunächst gesagt werden, daß dasselbe auf keinen Fall mit allgemeiner Gültigkeit richtig beurteilt werden kann, wenn ausschließlich die Verhältnisse der Unterextremität des Menschen maßgeblich sein sollen. Hier ist eine starke Störung eingetreten, welche sich in heftigen, Umbauvorgängen in Gestalt wiederholter starker Reduction und Neuanlage der inneren Spongiosa und in einem sehr friihzeitigen Ersatz der in to to konzentrischen Compactastruktur durch eine aus longitudinal verlaufenden Haversschen Säulen kundgibt und als deren Urheber ich den aufrechten Gang verantwortlich machen 


\section{F. A. M. W. Gełhardt, Die spezielle funktionelle Anpassung usw.}

möchte. Sieht man deshalb für einen vorläufigen Überblick von einer besonderen Bevorzugung des Menschen zunächst ab, so kommt man etwa zu folgendem Resultat: Die in toto konzentrische Struktur und das Prinzip des Körpers gleicher Festigkeit spielen eine entscheidende mechanische Rolle bis fast zum erwachsenen Zustande. Mit dem Festwachsen der Epiphysen ist der definitive Eintritt in eine Periode besiegelt, in welcher unter dem nunmehr viel deutlicheren Einflusse der funktionellen Anpassung der ja nunmehr ganz einheitlich gewordene Röhrenknochen, der infolgedessen auch nicht mehr die frïheren Spannungsverhältnisse aufweist, seiner Umwandlung in ein trajectorielles Fachwerk mehr und mehr entgegengeht. Dabei stehe ich auf dem Standpunkte, daß die Rolle, zu der es in bezug auf die Bewältigung der Gesamtsumme der mechanischen Spannungsverhältnisse dieses Fachwerk bringt, eine für den ganzen Knochen in verschiedenen Fällen sehr verschieden wichtige ist. Meiner Meinung nach spielt auch später für sehr viele massiv ausgebildete Knochen die kompakte Wand noch die Rolle der Hauptstütze, der gegenüber dem Spongiosainhalt der Enden wesentlich die Rolle einer verteilenden und federnden Aufnahmestelle für die Beanspruchung zufällt. (Also eine Art aus den mechanischen Verhältnissen sich ergebender "Arbeitsteilung " an Stelle des nach "Teleologie* schmeckenden »in groben Zügen« »vorangelegten späteren Gesamtbauplanes«.) In andern Knochen, und hier stehen die trajectoriell gut ausgebildeten der unteren Extremitäten des Menschen, des Elefanten, der Grabenden und andre viel umbauende an erster Stelle, tritt schließlich alles andre hinter dem trajectoriellen Fachwerk zurìck, und hier ist ohne Zweifel auch seine mechanische Bedeutung eine bei weitem überragende im völlig ausgebildeten Knochen. Sie wird dies um so mehr, je mehr unablässige ausgedehnte Umbauvorgänge ihr dazu Gelegenheit geben, und sie bleibt deshalb auch als letzter Rest übrig, wenn die senile Atrophie eine übermäßige Reduction des widerstehenden Materials hervorgebracht hat. Im übrigen steht nichts im Wege, sondern es ist sogar bei der hier so empfindlichen gestaltenden Reaktion auf die durch mechanische Beanspruchung hervorgerufenen lokalen Spannungsverhältnisse überaus wahrscheinlich, daß auch diese Fachwerke durch entsprechendes Dichtstehen und gesetzmäßige Dickenänderungen ihrer Einzelstuitzelemente zu jeder Zeit » Fachwerke gleicher Festigkeit « repräsentieren, wenn eine solche Bezeichnung im Sinne der »Körper gleicher Festigkeit« zulässig erscheint. 